\title{
Gruppen leiten mit dem Leipziger Prozessorientierungsmodell
}

\section{Strategische Orientierung in Gruppen- und Einzelarbeitsprozessen}

\section{Uwe Nowak}

Zusammenfassung In diesem Beitrag der Zeitschrift für Psychodrama und Soziometrie wird das Leipziger Prozessorientierungsmodell vorgestellt, welches eine strategische Orientierung in Gruppen- aber auch Einzelarbeitsprozessen ermöglicht. Grundidee ist, dass professionelles Handeln u. a. auf Hypothesen gestützt ist. Beschrieben wird ein zirkulärer Prozess, welcher, jeweils bezogen auf eine konkrete Situation mit dem „Wahr - Nehmen“ beginnt und der Evaluation abgeschlossen wird. Zeitlich unterschieden werden Perspektiven akuten, vorbereitenden und nachbereitenden Handelns. Das Modell ist sowohl im Kontext der Ausbildung „Psychodrama“ als auch für die Ausbildung ,Supervision und Coaching“, aber auch für jegliche therapeutische, beraterische und allgemein soziale Arbeit, hervorragend als Trainingsund Reflexionsinstrument geeignet.

Schlüsselwörter Affektregulation · Psychodrama · Prozessmanagement · Hypothesen · Introspektion · Interventionsplanung · Reflexionsinstrument · Wahrnehmungssteuerung $\cdot$ Zielbildung 


\section{Groups lead with the Leipzig process orientation model}

Strategic orientation in group and individual work processes

Abstract In this article from the the journal of psychodrama and sociometry a model is presented which enables strategic orientation in group as well as in individual work processes. The basic idea is that professional action is supported by hypotheses. A circular process is described, which, according to a concrete situation, starts with "perception" and ends with the evaluation. A temporal distinction is made between perspectives of acute, preparatory and follow-up action. The model is excellently suited as a training and reflection instrument both in the context of the training of "Psychodrama" and for the training of "Supervision/Coaching".

Keywords Affect regulation - Psychodrama - Management of processes · Hypotheses · Introspection · Intervention planning · Reflection instrument · Perception control $\cdot$ Objective setting

\section{Einleitung}

Im Rahmen von Beratung, Supervision und Therapie besteht hinsichtlich eines professionellen Vorgehens die legitime Erwartung, dass Affekte und daraus resultierende Handlungen nach Möglichkeit vermieden werden. Nach Nowotny (1972, S. 82) ,vermindern Affekte die Bewusstseinsklarheit und unterbinden die geordnete, zielgerichtete Führung“. Umgangssprachlich transportieren verschiedene Redewendungen den Charakter von Affekt über: „Er oder sie war außer sich!“ oder „Ich war nicht ganz bei mir.“ oder „Er oder sie rastete aus.“ In unserem Modell resultieren aus Affekten die Modi: Flucht oder Angriff oder Erstarrung.

Die Grundforderung nach hypothesengestütztem Handeln wird implizit und teilweise auch explizit in den meisten Modellen und Ausbildungen bezüglich der o.g. Formate formuliert.

Das Prozessorientierungsmodell (POM) resultiert aus der Gegenüberstellung verschiedener Handlungsqualitäten, wobei wir „Hypothesengestütztes Handeln“ ( $\mathrm{HH})$ und „Affektgesteuertes Handeln“ (AH) unterscheiden. Das Ergebnis dieser Betrachtung ist ein stark vereinfachtes, pragmatisch formuliertes Handlungsmodell, welches Professionalität fördert. Wir verwenden es als „Folie“ für Reflexion und Planung jeglicher Gruppenarbeit und dabei für vorbereitendes, akutes und auch nachbereitendes Handeln. Wir gehen davon aus, dass dadurch hypothesengestütztes Handeln konsequent realisiert und affektgesteuertes Handeln ausgeschlossen oder wenigstens dessen Auftretenswahrscheinlichkeit reduziert werden kann. Dazu sind jeweils konkrete Fragen zu beantworten; wer, wann, warum, mit welcher Hypothese und welchem Ziel, welche Intervention plant, umsetzt und welche Effekte entstanden sind.

Die Praxis von Psychodrama legt sehr oft ein hohes Augenmerk auf exzellente, kreative und lebendige Arbeit mit ProtagonistInnen, aber gruppendynamische Aspekte und Orientierung auf Prozessgeschehen und damit die Handlungsregulation von LeiterInnen wird eher vernachlässigt. So hat Grete Leutz in ihrem viel 
gelesenen Standardwerk „Psychodrama - Theorie und Praxis“ reichliche zwei Seiten für eine derartige Betrachtung eingeräumt und zum Thema Affektregulation und introspektive Wahrnehmung von LeiterInnen z. B. bemerkt: „,Der Psychodramaleiter wird während der Spielphase von keiner Übertragung des Protagonisten getroffen“ (Leutz 1974, S. 85). Später wird von anderen AutorInnen intensiver bezüglich der Rolle und Handlungssteuerung von LeiterInnen nachgedacht. Von Ameln (2009) beschreibt z. B. Handlungstechniken und fordert ein theoretisches Konzept, ,in dem sich Leitungsentscheidungen aus einer begründeten Interventionstheorie ableiten“ (v. Ameln 2009, S. 52) und betrachtet differenzierter die Wahrnehmungsdimension von Leitung exemplarisch für den Modus „Exploration“ (ebd. S. 145).

Das hier beschriebene Modell versucht nun auf der Metaebene, Momente von Handlungsregulation systematisch abzubilden und damit, wie der Name nahelegt, eine bessere Orientierung und Selbstreflexion für LeiterInnen zu ermöglichen. Es entspricht in der Relation von Theorie und Praxis ungefähr der von Habermas (1996, S. 99) beschriebenen „Idealen Sprechsituation“ - also einem anzustrebendem Zielzustand.

Die Psychodramapraxis ist stets auch mit Morenos Grundidee von Spontaneität und Kreativität zu verbinden. Und was Schacht (2010, S. 67) allgemein dazu schreibt - ,gewöhnlich gelingt uns die Alltagsbewältigung mit Hilfe verfügbarer Rollenkonserven. Letztere sind habituelle, eingeschliffene Handlungsstrukturen, die routinemäßig ablaufen, ohne dass wir uns bewusst darauf konzentrieren müssen." gilt selbstredend auch für PsychodramaleiterInnen in der Arbeit mit Gruppen. Spannend wird es dann, wenn Routinen nicht ,ausreichen“ und Fluktuation nicht mit entsprechender Attribution gedämpft werden können. Schacht (ebda., S. 68), findet: „Meist erfolgt dies anfänglich unreflektiert im Rückgriff auf weitere Rollenkonserven.“. Neben dem Aspekt ,unreflektiert“, welcher in unserem Verständnis (s. oben) mit Affektnähe assoziiert wäre, fehlt bei der beschriebenen Reaktion die anzustrebende Spontaneität, als adäquate Reaktion auf eine neue Situation.

Natürlich bedeutet Reflexion und Planung mit dem POM keinesfalls die Abstinenz oder Geringschätzung von intuitivem Handeln, welches gerade im Psychodrama nicht nur im Rahmen von Einzelarbeit gefragt ist. Es geht eher um ein Instrument, das darin unterstützt, neben Intuition auch Planung und Reflexion handlungswirksam einzusetzen.

\section{Das Prozessorientierungsmodell (POM)}

Das Prozessorientierungsmodell (POM) bildet einen zirkulären Prozess ab, bei dem der Ausgangspunkt als Phänomen bezeichnet wird. Nach der Wahrnehmung geht es darum Hypothesen zu erstellen, Ziele zu erarbeiten, entsprechende Interventionen (Mittel) zu finden und nach der Handlung im Rahmen von Evaluation entstandene Effekte einzuordnen (siehe Abb. 1).

Unsere Arbeit mit diesem Modell entspricht im Ansatz der von Burkart (2010, S. 208) formulierten Auffassung von „Dialogischer Introspektion“: „Man kann sich dementsprechend den Prozess der Interpretation und der Aneignung von Geschehen (Hervorhebung des Autors) als einen inneren Dialog vorstellen, in dem der Mensch 


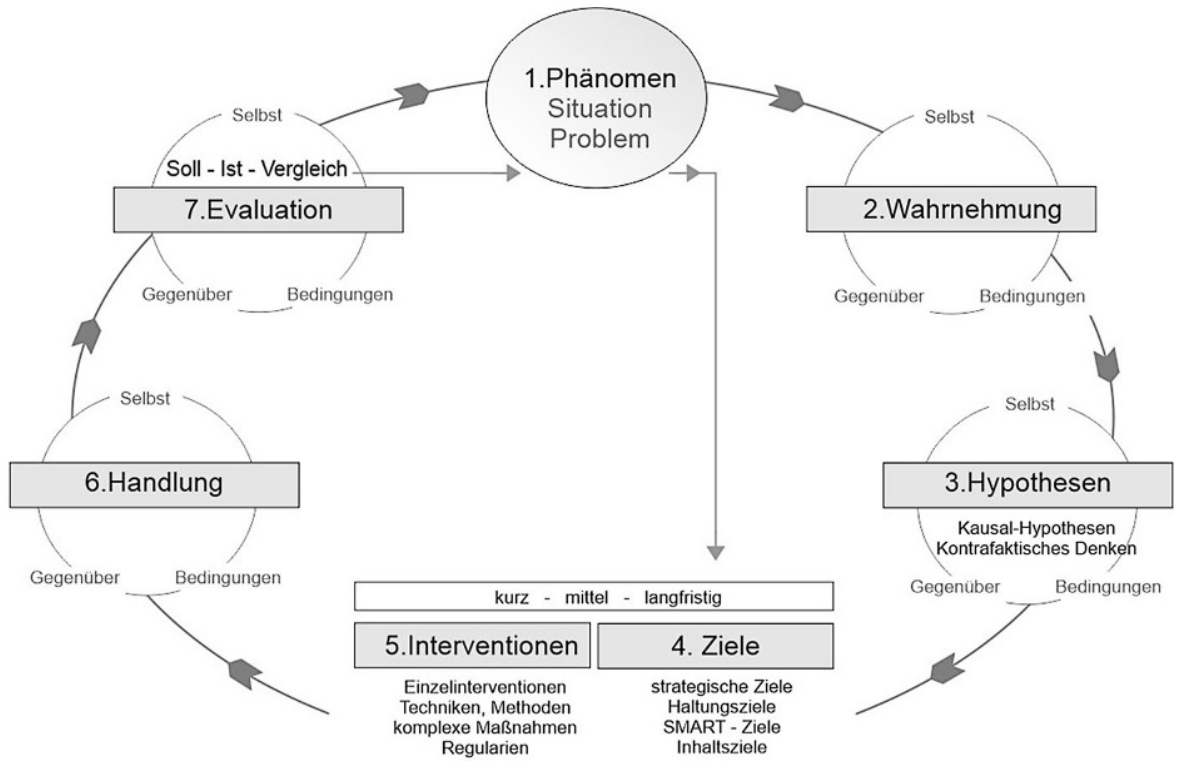

Abb. 1 Hypothesengestütztes Handeln und Leipziger POM (Nowak 2019)

die verschiedenen, aktuell bedeutsamen Perspektiven miteinander in Beziehung setzt und sie insbesondere im Hinblick auf ihre Konsequenzen miteinander vergleicht und daraus Schlüsse für weiteres Handeln zieht“.

Der Gesamtablauf eines Prozesses kann sich über Sekunden, Stunden oder Tage erstrecken. Je nach Dringlichkeit und zur Verfügung stehender zeitlicher Möglichkeiten wird das Modell also sehr schnell und reduziert oder ausführlicher und umfassender durchlaufen.

Neben der Beschreibung der Variante „Hypothesengestütztes Handeln“ soll auch kurz und skizzenhaft „Affektgesteuertes Handeln“ erörtert werden.

Begleitet wird dieses oft von einem höheren Stresspegel, hier auch verortet im Selbst, Gegenüber oder den Bedingungen. Was dazu führen kann, dass neben reduzierter Wahrnehmung unter massivem Verzicht auf differenzierte Hypothesen, mit Rückgriff auf Etikettierung und „Vereinfachung“ in einer Art „Affekt-Turbo“, ohne Ziel- und Interventionsabwägung schnell gehandelt wird. Und zwar mittels „Flucht“, „Angriff“ oder „Erstarrung“, (siehe Abb. 2).

Bei der Betrachtung beider Grafiken dürfte deutlich geworden sein, dass AH unter einer extremen Limitierung bezüglich Zeit, Perspektiven und Möglichkeiten abläuft und folgerichtig „Entschleunigung“ und umfassendere Orientierung uns davor bewahren hilft. Logischerweise sind unsere Handlungen neben dieser Limitierung immer auch inhaltlichen, ethischen und ,ideologischen“ (den jeweiligen „Schulen“ entsprechenden) Prinzipien unterworfen, worauf weiter unten im Abschnitt „Interventionen“ zum Thema „Widerstand“ kurz eingegangen wird. Psychodramatisch gedacht, sind es aber neben der Dimension der o.g. „Prinzipien“ eben auch unsere Rollenkonserven, welche durchaus als positiv regulierende, aber mitunter auch als spontaneitätshemmende Aspekte Wirksamkeit entfalten. 


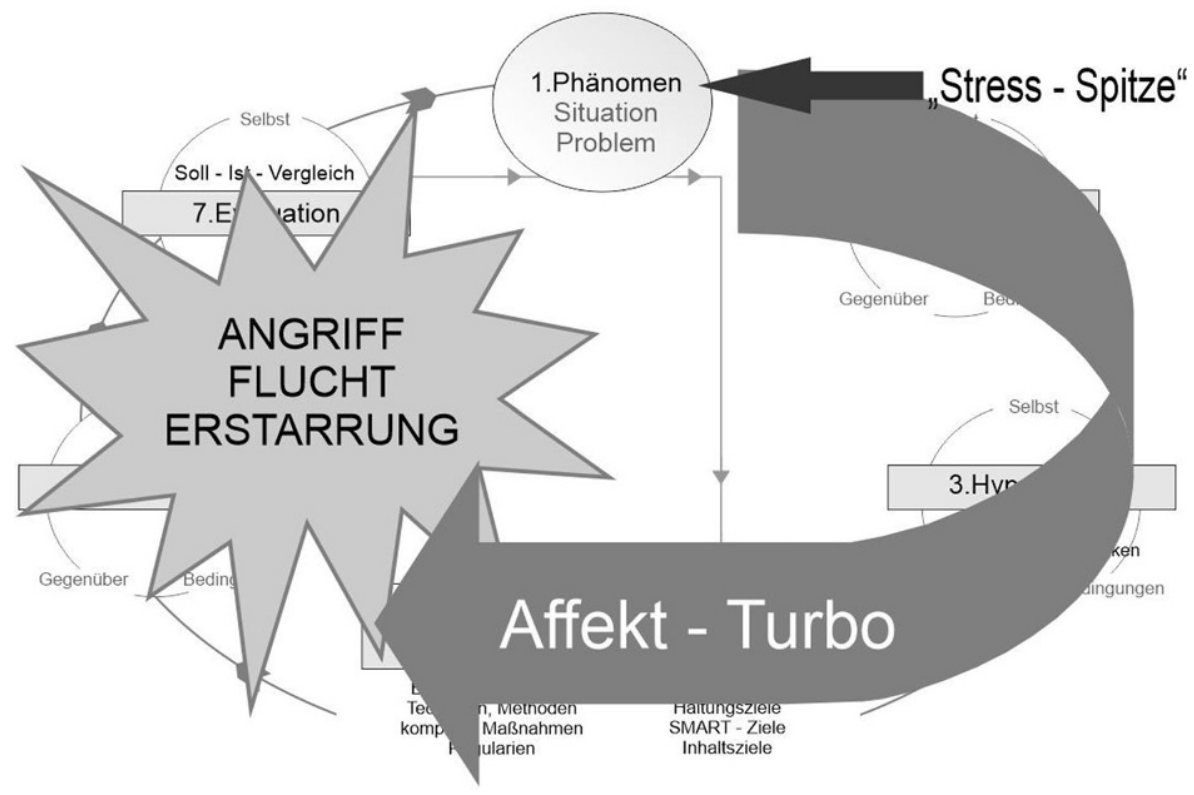

Abb. 2 Affektgesteuertes Handeln und Leipziger POM (Nowak 2019)

In den folgenden Abschnitten werden nun die einzelnen Elemente vom Leipziger Prozessorientierungsmodell (POM) vorgestellt, erläutert und anhand von Beispielen illustriert.

\subsection{Phänomen}

Verschiedene Konstellationen von Praxis werden hierbei erfasst und eine „Situation“, ein „Problem“, oder ein „Phänomen“ benannt bzw. betrachtet. Im weiteren Text verwende ich (der Einfachheit halber) immer den Begriff Phänomen. Wir unterscheiden Mikro- und Makrophänomene bezüglich Komplexität. So ist z. B. das Phänomen: „Teilnehmerin X hat in den ersten 30 min der Gruppenarbeit fünfmal gegähnt.“, eher überschaubar, während: „Ich führe einen Workshop zum Thema: Psychodramatische Arbeit im Rahmen von Coaching durch“, höhere Komplexität bedeutet.

Im Ausbildungskontext arbeiten wir sehr oft mit dem Modell und nutzen es als eine Art „Flugsimulator“ - wir beschreiben Phänomene und lassen die Studierenden den kompletten Zyklus durcharbeiten. Ein Beispiel: „Aus einer (eigentlich) spielfreudigen und gut angewärmten Gruppe findet sich niemand für eine Einzelarbeit.“ Dazu können jetzt alle einzelnen Elemente gedanklich erarbeitet werden, was, neben introspektiver Orientierung, Erklärungsansätzen und technischen Überlegungen zu mehr Sicherheit führt. Selbstverständlich für die psychodramatische Praxis ist das probeweise, szenische Realisieren, wobei die gewählten Ziele und Interventionen in einer Aktion ausprobiert und evaluiert werden können. 


\subsection{Wahrnehmung}

Bezogen auf ein Phänomen wird im ersten Schritt zunächst immer bewusste Wahrnehmung forciert; Was nehme ich bei mir selbst wahr (z. B. körperliche Anspannung, Ärger, Entspannung, Freude ...), was bei meinem Gegenüber (z. B. Stimmlage, Mimik, Anspannung ...) und was in Richtung der Bedingungen (z. B. Uhrzeit, Arbeitsbedingungen, Phase der Sitzung, Thema, erster Gruppentermin ...).

Bevor die nächste Intervention bzw. Handlung geplant und realisiert wird, geht es um ein umfassenderes Verstehen und Praktizieren von „Awareness“ im Sinne von Achtsamkeit. Sehr differenziert hat sich bereits Schacht (2010, S. 374) damit auseinandergesetzt und treffend formuliert: „Es geht darum sich von Erwartungen, Zielen, Absichten zu lösen.“ Ein Zugang, den wir explizit gerade für die Wahrnehmungsphase fordern. Was unter Umständen auch sehr herausfordernd sein kann, wenn LeiterInnen Phasen von Instabilität im Übergang zur kreativen Phase aushalten müssen. Das Eingreifen mittels vorschneller Aktionen oder Erklärungen könnte nämlich letztendlich Spontaneität verhindern, statt diese zu fördern.

Der im Psychodrama zentrale und hocheffektive „Rollentausch“ ist so auch mit dem „Subjekt“ Gruppe möglich und hilfreich. Insofern versuchen wir gerade bei Auszubildenden auch ein tieferes Verständnis dafür zu entwickeln, dass die Trennung von Wahrnehmung und Urteil (Hypothese) uns davor bewahren kann, vorschnell unangemessene Reaktionen zu vollziehen und inadäquate Entscheidungen (Interventionen) zu wählen - ähnlich dem journalistischen Prinzip, welches die „Trennung von Information und Meinung“ als qualitatives Kriterium beschreibt.

Selbstverständlich wird es immer ein Fokussieren auf einzelne Wahrnehmungsinhalte geben, wenn wir Komplexität reduzieren und die, natürlich nie fehlerfreie, Entscheidung über ,relevant“ oder ,irrelevant“ treffen. Klar ist dabei auch, dass je nach Stressniveau oder motivationaler, persönlicher Situation einzelne Aspekte von Wahrnehmung in unterschiedlicher Dominanz und Qualität erscheinen, siehe das Konzept „Tendenziöse Apperzeption“ von Adler, welches Datler (1995, S. 37) eingehend erläutert.

Darüber hinaus kann unsere Einstellung zum jeweiligen Gegenüber Einfluss auf unsere Wahrnehmungen und Handlungen haben, siehe die Ausführungen von Rosenthal und Jacobsen (1971, S. 294) zum „Pygmalion-Effekt“.

Beispiele von Wahrnehmung könnten sein:

- Wahrnehmung-Selbst: „Ich fühle Anspannung und leichten Ärger.“

- Wahrnehmung-Gegenüber: „Teilnehmerin X gähnt erneut.“

- Wahrnehmung-Bedingungen: „Der Arbeitsraum ist eng, warm und stickig.“

Die Wahrnehmungsinhalte werden im nächsten Schritt zur Grundlage für Hypothesen.

\subsection{Hypothesen}

Beim zweiten Schritt geht es, logischerweise orientiert an den Wahrnehmungsinhalten, um die Bildung von Hypothesen und dies auch wieder mit Einbeziehung von: Selbst, Gegenüber, Bedingungen. 
Was wir im Rahmen von Ausbildungsgruppen oft beobachten konnten ist, dass Auszubildende Hypothesen mit Gewissheiten gleichsetzen, was bei entsprechender Dynamik leider häufig zu sich selbsterfüllenden Prophezeiungen führt. Notwendigerweise sei hier also mit Hilfe von Roscher (2009, S. 2) kurz auf den Unterschied hingewiesen: „Hypothesen sind eine Unterform von Thesen: während eine These eine einfache Behauptung darstellt, behauptet eine Hypothese einen Zusammenhang zwischen mindestens zwei Faktoren; etwas zugespitzter ist sie die Vermutung einer Ursache-Wirkungsbeziehung, welche sich in Wenn-Dann- oder Je-Desto-Aussagen formulieren lässt.“.

Dies ist wichtig, wenn wir einbeziehen, dass wir uns bezüglich unserer Hypothesen eben auch irren können und wir uns Gewissheiten erst, oft dialogisch, erarbeiten müssen.

Grundsätzlich werden Hypothesen je nach Bedarf und Lage retrospektiv, prospektiv oder bezogen auf das „Hier und jetzt“ orientiert sein. Beispiele für Hypothesen könnten sein:

Hypothese-Selbst: Ich bin angespannt und ärgerlich, weil Teilnehmerin $\mathrm{X}$ sich langweilt.

Hypothese-Gegenüber: Teilnehmerin X gähnt, weil sie eine harte Woche hinter sich hat.

Hypothese-Bedingungen: Teilnehmerin X gähnt, weil zu wenig Sauerstoff im Raum ist.

Bei Makrophänomenen werden wir oft umfänglich Konzepte und Konstrukte zur jeweiligen Praxis als Hypothesen einbeziehen. So kann ich zum Beispiel in einer Gruppensituation von „Schwere“, „Stagnation“ oder ,aggressivem Klima“ das Modell von Tuckman (1965) zu „Gruppenphasen“ (forming, storming, norming, performing) aktivieren und erwägen: „Okay, die Gruppe reagiert so, weil die ,storming Phase" noch nicht abgeschlossen ist." Wird das POM vorbereitend erarbeitet, werden meist abstrahierte und auch erfahrungsbezogene Hypothesen aufgestellt, z. B. für den o.g. Workshop „Psychodramatische Arbeit im Rahmen von Coaching“ in der Form: „Aufgrund unterschiedlicher Vorerfahrungen bzgl. der Methode Psychodrama werden TeilnehmerInnen bei Begrifflichkeiten und Techniken mehr oder weniger Erklärungen brauchen.“ oder für einen Gruppenprozess einer Selbsterfahrungsgruppe: „Weil die Gruppe bei dem letzten Termin ablehnend wegen der bewegungsorientierten Übung B2 reagiert hat, könnte es sein, dass ich generell zu vorsichtig werde, bezogen auf den Einsatz solcher Übungen."

Einige LeserInnen werden an dieser Stelle fast zwangsläufig das Modell „KausalAttribution" von Heider (1977, S. 84) aktivieren, welches wir bei differenzierten Analysen v.a. in der Dimension des Selbst durchaus einbeziehen.

Neben Kausalhypothesen ist auch das von Kahnemann beschriebene Konstrukt „Kontra-faktisches Denken“ (ausführlicher erläutert in Aronson et al 2004, S. 90) sehr gut geeignet, Phänomene retrospektiv differenzierter zu analysieren, Nach dem Prinzip ,what would have to be different?“ wird dabei, entgegen der stattgefundenen 
Praxis, nach alternativen Szenarien und Ansätzen gefragt. So könnte nach dem letztgenannten Beispiel jemand kontrafaktisch feststellen: „Wenn ich mir mehr Zeit bei der Instruktion der Übung B2 genommen hätte, wären wahrscheinlich mehr TeilnehmerInnen zu begeistern gewesen." Hier werden übrigens, was bei kontrafaktischem Denken oft vorkommt, auch schon Ziele formuliert, welche den nächsten logischen Schritt im Zyklus des POM's betreffen.

Auch bei der Er- und Bearbeitung von Hypothesen muss eine Auswahl erfolgen; ich kann in einer konkreten Situation nicht bezüglich aller Wahrnehmungsinhalte massenhaft Hypothesen bilden, sondern reduziere im Modus von (Stress)Regulation immer Komplexität und werde dann das jeweilige Ziel erstellen. In akuten Situationen, welche schnelles Handeln erzwingen, wird sich das entsprechend zeigen. Erinnert sei an das Wort von Ruth Cohn: ,never analyze in a burning house“ (Farau und Cohn 1984, S. 361).

\subsection{Ziele}

Was soll, muss als Nächstes passieren? Was will ich? Was braucht es?

Wir plädieren hier für eine klare Trennung von Ziel und Intervention, damit ein hohes Maß an Freiheit bezüglich der Zielformulierung erhalten bleibt und nicht eine Limitierung durch die jeweils intern abgebildeten, vorhandenen Interventionsmöglichkeiten stattfindet. Viele von uns kennen die oft verwendete Metapher, bei welcher sich nur ein Hammer im Werkzeugkasten befindet und deshalb alles wie ein Nagel ,behandelt“" werden muss.

Erstens lassen sich, wenn ich frei in der Zielformulierung bleibe, häufig auch ,adaptierte Werkzeuge" suchen und finden. Zweitens kann, bei einem veröffentlichten und gemeinsam validierten Ziel und ,fehlender“ Intervention, das jeweilige Gegenüber selbstwirksam und aktiv eigene Interventionen vorschlagen und so partizipativ bei der Zielerreichung tätig sein. Bezogen auf das Konzept „Kreativer Zirkel“ (Hutter 2000 zitiert nach v. Ameln 2009, S. 209) muss es für LeiterInnen darum gehen, sensibel die entsprechenden Übergänge (... Erwärmung, Spontaneitätslage, Kreative Phase ...) wahrzunehmen und nicht vorschnell per Definition oder Intervention zu „überdecken“.

Generell strukturieren wir Überlegungen zu diesem Bereich mit der Unterscheidung von strategischen Zielen, Haltungszielen, SMART-Zielen (Doran 1981, S. 35) und inhaltlichen Zielen.

Strategische Ziele generieren wir mit Hilfe des Modells der „Interventions-Pole“ (Nowak, unveröffentlichtes Lehrmaterial vom PDI Leipzig), bei dem auf verschiedenen Skalen unter-schiedliche Qualitäten abgebildet werden, z. B. stehen sich die Pole „Detail“ und „Ganzes“ gegenüber. Zunächst wird auf dieser Skala die aktuelle Position verortet und danach überlegt, was für das konkrete Phänomen angemessen bzw. notwendig ist, z. B. eine mehr am „Detail“ oder am „Ganzen“ orientierte Strategie.

Bezüglich der ,gähnenden Teilnehmerin X“ könnte also hier ein strategisches Ziel lauten: „Ich nehme die gesamte, sehr aktive und interessierte Gruppe (Ganzes) in den Blick.“ Möglich auch: „Ich beobachte X (Detail) genauer und erkunde, ob sie sich langweilt.“ 
Haltungs- und Mottoziele werden mit dem Zürcher Ressourcenmodell (Storch und Krause 2007, S. 92) in einem Modus von „Selbstmanagement“ erarbeitet. Sie können durch Selbstaufmerksamkeitslenkung, u.a. mit der Registrierung ,,somatischer Marker" (Damasio 2001, S. 77) aktualisiert werden und haben gegenüber Verhaltenszielen ein breiteres Wirkungsspektrum.

Das Konzept der SMART-Ziele, welches in der Regel Verhaltensziele zu erarbeiten hilft, ermöglicht ebenfalls eine strukturierte Herangehensweise in Richtung Zielfindung. Es soll aber hier (auch wegen des hohen Grades an Bekanntheit) nur kurz erwähnt werden. Ein auf die Bedingungen orientiertes Ziel bezüglich der Teilnehmerin X (bei vermuteter „Hypoxie“) wäre: „Ich sorge jetzt für Frischluft!“

Inhaltliche Ziele sind oft durch das jeweilige Arbeitsformat, aber auch durch thematische „Festlegung“ vorbestimmt. So wäre in dem o.g. Workshop „Psychodramatische Methoden ..." das Ziel ,Vorstellen und trainieren von psychodramatischen Techniken“ inhaltlich angemessen, aber „Vermittlung von Moderationstechniken“ eher deplatziert.

Für eine der oben aufgeführten Hypothesen „storming Phase“ des Makrophänomens „Gruppensituation“ könnte folgendes Zielbeispiel dienen: „Ich werde die Gruppe darin unterstützen, die ,norming Phase " einzuleiten.“

Nach der Hierarchisierung und Entscheidung bezüglich relevanter, akuter, langoder mittelfristiger Ziele, führt der nächste Schritt zu den Interventionen.

\subsection{Interventionen}

Wenn klar ist, was angestrebt wird, muss über die entsprechenden, praktikablen und zur Verfügung stehenden oder zu organisierenden Mittel (Interventionen) nachgedacht werden.

Bei sehr trivialen und naheliegenden Verknüpfungen von Ziel und Intervention sprechen wir von Einzelinterventionen. Passend ist hier das Beispiel mit dem Ziel: „Frischluft!“ und der Intervention: „Fenster öffnen!““ oder das Beispiel mit dem Ziel: „Ich erkunde, ob X sich langweilt.“ und der Intervention: „Ich frage X, ob sie sich langweilt oder ich frage nach einem inhaltlichen Aspekt meiner letzten Ausführung."

Daneben gibt es jedoch wesentlich häufiger Verknüpfungen, welche komplexe Maßnahmen und mehrstufige Interventionen erforderlich machen, was im nächsten Kapitel in Ausschnitten illustriert wird.

Selbstverständlich nutzen wir an dieser Stelle stets auch bekannte und stabil verknüpfte Varianten von Zielen und Interventionen, welche teilweise automatisiert sind und auch durchaus als regelrechte Stressroutinen vollzogen werden. So wird z. B. in einer unstrukturierten, unübersichtlichen Gruppensituation das strategische Ziel „Mehr Struktur“ mit der Intervention (Methode) ,Visualisierung“ einhergehen oder in einer eskalierten, aggressiven Situation das Ziel ,Entschleunigung“ mit der Intervention: „Pause“ oder „Kommunikation mittels kontrolliertem Dialog“ angestrebt.

Und natürlich bestimmen die weiter oben bereits erwähnten „Schul-Prinzipien“ und die ebenfalls schon erwähnten „Rollenkonserven“ unser Denken und Handeln bei der Abwägung von Zielen und Interventionen. Betrachten wir exemplarisch das Phänomen „Widerstand“, so zeigen sich dabei relativ stabile Verknüpfungen, wenn wir die unterschiedliche Positionierung psychotherapeutischer Schulen anschauen. 
Petzold (1985, S. 7) hat dies systematisch und umfassend dargestellt, indem er für einige Therapierichtungen das Zusammenwirken von Hypothesen, Zielen und Interventionen gegenüberstellt.

Die im jeweiligen Repertoire vorhandenen Methoden und Techniken eröffnen dabei weiträumige oder eingeschränkte Kombinationsmöglichkeiten. So können LeiterInnen, die mit einer Gruppe vorwiegend im Kommunikationsmodus „Diskussion“ (Buer 2004, S. 18 ff.) arbeiten, das Ziel „Exploration der Gruppendynamik“ nur dann mit der Intervention „Aktions-Soziometrie“ umsetzen, wenn sie über diese Methoden und Techniken verfügen und die entsprechende Anwendungssicherheit entwickelt haben.

In der schon weiter oben bemühten Metapher bedeutet dies, dass ich ohne Hammer wohl auf das Einschlagen eines Nagels verzichten muss und ohne Zange einen „falsch platzierten Nagel“ eben nicht mehr zu entfernen versuche. Das bedeutet schließlich, dass PsychodramatikerInnen stets angehalten sind, eigene Entwicklung und ständiges Lernen als lustvolle Aufgabe zu begreifen. Etwas, was Zerka Moreno übrigens einmal in einer unserer Trainingsgruppen in Beacon als ,Jungbrunnen für Herz und Kopf" beschrieb, was für sie unschwer zu belegen wäre: sie wurde fast 100 Jahre alt.

Regularien sind Interventionen, welche einmal stabil installiert, zu einem „Kulturelement“ werden können, Beispiel sei der „Redestein“, welcher in Kindergruppen (und auch bei ausufernden Erwachsenen) eine Struktur schafft, mit welcher Redezeit verteilt wird. Derartige Regeln und deren ,Symbole“ können später überflüssig oder auch diskutiert und wieder abgeschafft werden.

\subsection{Handlung}

Nach der Entscheidung, die intern gefällt und/oder mit dem jeweiligen Gegenüber ausgehandelt wurde, welche Interventionen realisiert werden, folgt die eigentliche Handlung, nämlich die aktive, tätige Umsetzung der jeweiligen Interventionen.

Vor allem in komplexen Situationen betonen wir an dieser Stelle immer den Charakter von „Experimenten“ und nähern uns gemeinsam demütig sozialen Feldern mit dem viel zitierten Motto: „Wir irren uns voran“ von Odo Marquard (Marquard 2002 zitiert nach Sprenger 2002, S. 199). Was als Haltung m. E. eben weniger (wie immer mal vermutet) einem Glücksspiel, sondern mehr einem gerichteten, planvollen Handeln entspricht, welches aber auf das Dogma und den Zwang „Goldener Weg“ verzichtet und so „Fehlerfreundlichkeit“" und eine Neuorientierung offen hält.

\subsection{Evaluation}

Der letzte Schritt vergleicht die Effekte der gewählten umgesetzten Interventionen mit den angestrebten Zielen bezogen auf das Ausgangsphänomen und stuft das Ergebnis als entweder umfänglich oder teilweise erfolgreich ein. Daraufhin beginnt ein neuer Prozess bezüglich der nun entstandenen Situation.

Für diese Phase soll noch einmal Schacht (2010, S. 82) zitiert werden, wenn er für das „Ideal des spontan-kreativen Handelns“ vorschlägt: „Das bedeutet, Ziele einer- 
seits engagiert und entschlossen, andererseits jedoch mit spielerischer Leichtigkeit zu verfolgen. Es sollte sich um imperfekte Ziele handeln.“

Das bildet unsere Haltung zu Kreativität, Lebendigkeit und dialogischer Gruppenleitung (im Innen und Außen) sehr gut ab; es geht weniger darum, das ,genau Richtige“ zu tun, sondern mehr um Klarheit und Gerichtetheit. Ich kann, darf, werde mich in meinen Entscheidungen und Vermutungen auch irren. Aber ich werde dabei auf einem Weg sein, der mich jeweils weiterführen wird und durch bewusste Erfahrung wachsen und im klassisch psychodramatischen Verstehen, spontaner werden lässt.

\section{Ein Praxisbeispiel}

Im folgenden Kapitel wird eine Arbeitssequenz aus einer Psychodrama-Selbsterfahrungsgruppe vorgestellt und dabei versucht, den gesamten Zyklus des erläuterten Modells sichtbar zu machen. Deutlich wird auch, dass bei unterschiedlichen Szenarien (z. B. „Einzelintervention“ oder „Komplexe Maßnahme“) bezüglich der Planung konkret mit der Zeitdimension gearbeitet werden muss und logischerweise sowohl die notwendigen Bedingungen als auch das „Mandat“ für entsprechende Interventionen vorliegen sollten.

Phänomen: In einer Selbsterfahrungsgruppe wird bei einer Skalierung mit der Frage nach bisherigen Einzelarbeiten deutlich, dass Lukas (Name geändert) bisher noch keine einzige Protagonistenarbeit auf der Bühne hatte.

Wahrnehmung: Bei dem kurzen „Skalen-Interview“ wirkt Lukas sehr betrübt und beschämt (Wahrnehmung-Gegenüber), die anderen TeilnehmerInnen (TN) zeigen nonverbal mitfühlende, aber auch distanzierte Reaktionen. Ich selbst spüre neben Mitgefühl auch Neugier (Wahrnehmung-Selbst). Die Gruppe steht bei 4 und 3 auf der Skala, eine Teilnehmerin bei 2 (Wahrnehmung-Bedingungen).

\section{Hypothesen:}

Hypothese-Gegenüber 01: Lukas fürchtet sich aufgrund eines vorhandenen, aber schambesetzten Themas vor der Arbeit auf der Bühne und ist unsicher, wie die Gruppe reagiert, ist also in der Phase der „Erwärmung“ und in seiner Spontaneität beeinträchtigt.

Hypothese-Gegenüber 02: Die Gruppe ist bezüglich der Reaktion heterogen und eventuell für Lukas nicht stabil genug sichtbar.

Hypothese-Gegenüber 03: Scham führt zu Rückzug und ist soziometrisch potenziell isolierend.

Hypothese-Bedingungen 01: Die Gruppe ist arbeitswillig und zugewandt.

Hypothese-Bedingungen 02: Es ist der erste Tag eines dreitägigen Seminars, also kein Zeitdruck und damit auch ein tragfähiger Rahmen.

\section{Ziele:}

Ziel-Gegenüber 01: Lukas bekommt im „Hier und Jetzt“ einen differenzierten Blick auf die Gruppensituation und erlebt sich als wichtigen, angenommenen Teil der Gruppe. 
Ziel-Gegenüber 02: Einzelne TN, welche sich im Modus projektiver Ablehnung befinden, erkennen eigene „Schwierigkeiten“ sich zu zeigen an.

Ziel-Gegenüber 03: Lukas wird ermutigt, mittelfristig (u.U. auch kurzfristig) in einer neuen Spontaneitätslage eine Einzelarbeit zu wagen.

Die gewählten Interventionen und Handlungen verfolgen mehrstufig alle drei Ziele.

Intervention 01: Die Gruppe wird aufgefordert sich ein Requisit zu suchen, welches für eigenes, bisher noch nicht in der Gruppe gezeigtes, schambesetztes Thema steht. (Es wird vorher explizit zugesichert, dass das Thema selbst nicht benannt werden muss.)

Intervention 02: Alle TN arbeiten zunächst angeleitet, mit einem leeren Stuhl als Gegenüber, im stillen Dialog: „Ich, das Thema spreche zu Dir und umgekehrt." (Hier geht es darum, emotionale Nähe zum Thema aufzubauen, welche für den nächsten Schritt wichtig ist.)

Intervention 03: Alle TN stehen im Kreis und legen nacheinander das Requisit in die Mitte und beenden den Satz: „Wenn Ihr das über mich wissen würdet, wünschte ich mir von Euch ...". (Wichtig dabei ist, dass Lukas im Uhrzeigersinn zuletzt im Kreis steht, das bedeutet, die Gruppe geht jetzt in „Vorleistung“.) Psychodramatische KollegInnen werden hier unschwer ein ,,vorgezogenes Sharing“ entdecken. Angestrebt wird das, was wir mit „Sharing“ erreichen können, nämlich die (Re-)Integration eines Gruppenmitglieds, welches sich (in der Regel durch Arbeit auf der Psychodramabühne) exponiert und damit von der Gruppe ein Stück entfernt hat. Mir ist dabei wichtig, dass für die ProtagonistInnen jeweils gut nachvollziehbar wird, dass es sich um ein wirkliches „Sharing“ handelt, was durch Intervention 02 erreichbarer wird.

Illustrieren möchte ich den eingetretenen Effekt mit zwei Sätzen aus der Gruppe, so ergänzte ein Teilnehmer den Satz mit ,... wünschte ich mir von Euch, dass ich nicht darauf reduziert werde, ich bin mehr als das hässliche, schreckliche Etwas.“ Und eine Teilnehmerin vollendete den Satz mit ,... wünschte ich mir, dass wir nach vielen Tränen und Betroffenheit auch wieder gemeinsam lachen können.“

Evaluation: Lukas signalisiert im Anschluss an die Übung große Dankbarkeit in Richtung Gruppe, beschreibt ein tiefere Ahnung von ,,Verbundenheit“ und nimmt sich vor, am nächsten Tag eine Einzelarbeit zu einem ,einfachen Thema“" zu wagen. Was nicht am nächsten Tag, aber beim nächsten Gruppentermin in der Tat so wird.

Nach der Erstvorlage dieses Beitrages wurde ich von der Redaktion gebeten, ergänzend auch etwas über Stärken und Schwächen des Modells zu schreiben.

Als Praktiker arbeite ich (wie inzwischen auch unsere Auszubildenden) sehr gern mit dem Modell, weil es hilfreich ist, in komplexen Prozessen eine Orientierung zu bekommen und retrospektiv im Rapportmodus auch etwas über vorschnelle „Lösungen“ oder „Vorlieben zu erkennen“. Es ruft auch ins Gedächtnis, dass jegliche „Theorie“ und „Folie“ immer wieder auf den Prüfstand gehört. Im Psychologiestudium wurden wir diesbezüglich früh zu einer demütigen Balance von Theorie und Praxis ermutigt. Die zusammengefasste These von A. N. Leontjev (zitiert nach 
Schartmann 1998, S. 53) „Die Tätigkeit ist immer reicher als das Abbild“ weist m. E. darauf hin, dass wir mit Modellen sozusagen immer hinterherlaufen.

Ähnlich der aktuellen Situation mit dem Corona-Virus, wenn wir etwas entdeckt haben werden, was diesbezüglich (möglicherweise) hilfreich ist, wird uns die Tätigkeit, das Leben, vor neue Herausforderungen, neue „Viren“ stellen.

Wohl ist das eine klar benennbare Grenze meines Modells. Es gibt lediglich einen kleinen Ausblick auf einige Möglichkeiten, aber ist bei Weitem nicht in der Lage, Wirklichkeit und Praxis umfassend abzubilden. Treffender formuliert von Marx und Engels (1977, S. 825) ,,... alle Wissenschaft wäre überflüssig, wenn die Erscheinungsform und das Wesen der Dinge unmittelbar zusammenfielen“.

\section{Literatur}

Ameln, F. v. (2009). Psychodrama. Heidelberg: Springer.

Aronson, E., Wilson, T., \& Akert, R. (2004). Sozialpsychologie. München: Pearson Studium.

Buer, F. (2004). Praxis der Psychodramatischen Supervision. Wiesbaden: VS.

Burkart, T. (2010). Dialogische Introspektion. Wiesbaden: VS Springer.

Damasio, A. (2001). Ich fühle, also bin ich. München: List.

Datler, W. (1995). Tendenziöse Apperzeption. In R. Brunner., M. Tilze (Hrsg.), Wörterbuch der Individualpsychologie.

Doran, G. (1981). There's a S.M.A.R.T. way to management's goals and objectives. Management Review, $70,35-36$.

Farau, A., \& Cohn Ruth, C. (1984). Gelebte Geschichte der Psychotherapie. Stuttgart: Klett-Cotta.

Habermas, J. (1996). Moralbewußtsein und kommunikatives Handeln. Frankfurt a.M.: Suhrkamp.

Heider, F. (1977). Psychologie der interpersonalen Beziehungen. Stuttgart: Klett.

Leutz, G. (1974). Psychodrama. Theorie und Praxis. Berlin Heidelberg New York: Springer.

Marx, K., \& Engels, F. (1977). Werke. Bd. 25. Berlin: Rosa Luxemburg Stiftung.

Nowotny, E. (1972). Psychologie Einführung und Übersicht. Wien: Eugen Ketterl.

Petzold, H. (1985). Widerstand. Ein strittiges Konzept in der Psychotherapie. Paderborn: Junfermann.

Roscher, K. (2009). Hinweise zur Formulierung von Thesen, Hypothesen und Annahmen. online

Rosenthal, R., \& Jacobsen, L. (1971). Pygmalion im Unterricht. Weinheim: Beltz.

Schacht, M. (2010). Das Ziel ist im Weg. Wiesbaden: VS.

Schartmann, D. (1998). Persönlichkeitsfördernde Arbeitsgestaltung mit behinderten Menschen. Münster: LIT.

Sprenger, R. (2002). Prinzip Selbstverantwortung. Frankfurt, New York: Campus.

Storch, M., \& Krause, F. (2007). Selbstmanagement - ressourcenorientiert. Bern: Huber.

Tuckman, B.W. (1965). Developmental sequences in small groups. Psychological Bulletin, 63, 384-399.

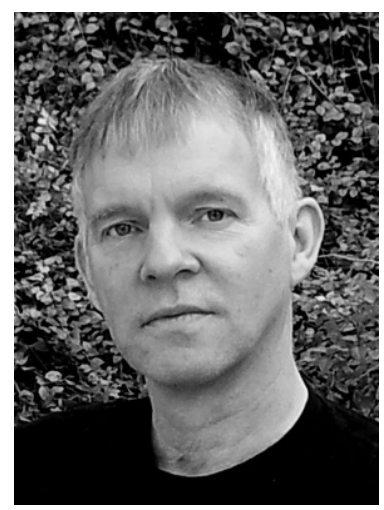

Uwe Nowak 1959, Diplom Psychologe (Klinische Psychologie) Mitbegründer und Ausbildungsleiter vom Psychodrama Institut Leipzig (www.pdi-leipzig.de), Leiter vom IPP Leipzig (www.ipp-leipzig.de), Supervisor (BDP) und Coach, zertifizierter ZRM-Trainer, freiberuflich tätig. 